\title{
WEAKLY COMPACT CARDINALS AND NONSPECIAL ARONSZAJN TREES
}

\author{
SAHARON SHELAH AND LEE STANLEY
}

(Communicated by Thomas J. Jech)

\begin{abstract}
.
LEMMA 1. If $\lambda$ is a cardinal with $\mathrm{cf} \lambda>\omega$, then $\square_{\lambda}$ implies that there is a $\lambda^{+}$-Aronszajn tree with an $\omega$-ascent path, i.e. a sequence $\left(\bar{x}^{\alpha}: \alpha<\lambda^{+}\right)$with each $\bar{x}^{\alpha}=\left(x_{n}^{\alpha}: n<\omega\right)$ a one-to-one sequence from $T_{\alpha}$, such that for all $\alpha<\beta<\lambda^{+}$, $x_{n}^{\alpha}$ precedes $x_{n}^{\beta}$ in the tree order for sufficiently large $n$.

LEMMA 2. If $\lambda$ is a cardinal with $\operatorname{cf} \lambda=\omega<\lambda$, then $\square_{\lambda}$ implies that there is a $\lambda^{+}$-Aronszajn tree with an $\omega_{1}$-ascent path (replace $\omega$ by $\omega_{1}$, above).

LEMMA 3. If $\lambda$ is an uncountable cardinal, $\kappa$ is regular, $\kappa<\lambda$, cf $\lambda \neq \kappa, T$ is a $\lambda^{+}$-Aronszajn tree, and $\left(x_{i}^{\alpha}: i<\kappa\right)$ is a one-to-one sequence from $T_{\zeta(\alpha)}$ with the property of ascent paths, where $\varsigma: \lambda^{+} \rightarrow \lambda^{+}$is a monotone increasing function of $\alpha$, then $T$ is nonspecial.

THEOREM 4. If $\lambda$ is uncountable, then $\square_{\lambda}$ implies that there is a nonspecial $\lambda^{+}$-Aronszajn tree.

THEOREM 5. If $\lambda$ is an uncountable cardinal, $\kappa=\lambda^{+}$, and $\kappa$ is not (weakly compact $)^{L}$, then there is a nonspecial $\kappa$-Aronszajn tree.
\end{abstract}

Notation. $\mathrm{CH}$ is the continuum hypothesis. Souslin trees are $S$-trees; $\kappa$-Souslin trees are $\kappa$ - $S$-trees. Aronszajn trees are $A$-trees; $\kappa$-Aronszajn trees are $\kappa$ - $A$-trees. A $\kappa$-wide tree is a tree of height $\kappa$ with node set a subset of $\kappa$, with no $\kappa$-branches. $\mathrm{SH}_{\kappa}$ is the $\kappa$-Souslin Hypothesis: there are no $\kappa$-Souslin trees. If $\kappa=\lambda^{+}$and $T$ is a $\kappa$-wide-tree, then $T$ is $\kappa$-special iff there is $f: T \rightarrow \lambda$, such that for any chain $C$ of $T, f \mid C$ is one-to-one (a more general formulation of this allows us to generalize the notion to regular limit cardinals, but we have no use for the generalization here). $\mathrm{SAH}_{\kappa}$ is the $\kappa$-special-Aronszajn Hypothesis: all $\kappa$ - $A$-trees are $\kappa$-special. Thus, $\mathrm{SAH}_{\kappa} \Rightarrow \mathrm{SH}_{\kappa}$. BA is Baumgartner's Axiom and BACH is the conjunction of BA and $\mathrm{CH}$; see [8 and 9, or 10]. Other notation will be introduced as needed or is intended to be standard or else have a clear meaning.

Introduction. In [7] we proved that $\mathrm{CH}+\mathrm{SH}_{\aleph_{2}}$ is a large cardinal hypothesis: we obtained consistency strength at least that of the existence of an inaccessible (we will write inaccessible $\leq$ cons $\mathrm{CH}+\mathrm{SH}_{\aleph_{2}}$, and similar expressions; equiconsistency statements will use $\sim_{\text {cons }}$ ). In [4], Laver and Shelah showed that $\mathrm{CH}+\mathrm{SH}_{\aleph_{2}} \leq$ cons weakly compact; in fact their methods show that $\mathrm{BACH}+\mathrm{SAH}_{\aleph_{2}} \leq$ cons weakly

Received by the editors July 16, 1986 and, in revised form, March 10, 1987.

1980 Mathematics Subject Classification (1985 Revision). Primary 03E05, 03E45, 03E55, 03E99, 04A20, 04A99.

The first author was partially supported by a BSF grant. Both authors were partially supported by NSF grants. 
compact. A natural conjecture, to which we are committed, is that $\mathrm{CH}+\mathrm{SH}_{\aleph_{2}}$ $\sim_{\text {cons }}$ weakly compact.

We obtained partial results in this direction in $[8,9]$ by showing that Mahlo $\leq$ cons $\mathrm{BACH}+\mathrm{SH}_{\aleph_{2}}$, improving an earlier result of Gregory that Mahlo $\leq$ cons $\left(\mathrm{CH}+2^{\aleph_{1}}=\aleph_{2}+\mathrm{SH}_{\aleph_{2}}\right.$ ) (to our knowledge, no upper bound for the consistency strength of this theory has been found). Here, we obtain a different kind of partial result. We show (Theorem 5 ) that $\mathrm{CH}+\mathrm{SAH}_{\aleph_{2}} \sim_{\text {cons }}$ weakly compact (of course, by [4] we need only show that weakly compact $\leq_{\text {cons }} \mathrm{CH}+\mathrm{SAH}_{\aleph_{2}}$, which is a contrapositive of Theorem 5). We should note that in the same spirit (a nonspecial $\kappa$ - $A$-tree is a poor man's $\kappa$ - $S$-tree) Lemmas 1 and 2 can be viewed as improvements in one dimension (weakening the hypotheses on cardinal exponentiation) but weakenings in another dimension (poor man's $\kappa$ - $S$-trees in place of real ones) of the result of Gregory et al. that, e.g., $\square_{\aleph_{1}}+\mathrm{CH}+2^{\aleph_{1}}=\aleph_{2}$, yield an $\aleph_{2}$-S-tree.

We are grateful to the referee for pointing out several inaccuracies in early versions of Lemmas 1 and 2 and their proofs. We are also grateful to S. Todorcević for many helpful discussions and for pointing out how to obtain an earlier version of Theorem 5 from a weaker version we had proved. This and other historical points will be discussed below.

1. Lemma 1. Throughout this section we are assuming that $\mathrm{cf} \lambda>\omega$ and that $\left(C_{\delta}: 0<\delta=\bigcup \delta<\lambda^{+}\right)$is a $\square_{\lambda}$-sequence. We shall construct a nonspecial $\lambda^{+}-A$ tree. We may assume (see [1, IV, Lemma 2.10]) that $S \subseteq\left\langle\delta<\lambda^{+}: \operatorname{cf} \delta=\omega\right\rangle, S$ is stationary in $\lambda^{+}$but that, for all positive limits $\delta<\lambda^{+}, C_{\delta}^{\prime} \cap S=\varnothing$ (recall that $C_{\delta}^{\prime}$ is the set of all limit points of $C_{\delta}$ except $\delta$ ).

We construct $T|\alpha=(T \mid \alpha, \triangleleft), f| \alpha=_{\text {def }} f \mid(T \mid \alpha)$, and $\left(\bar{x}^{i}: i<\alpha\right)$ by recursion on $\alpha<\lambda^{+}$, where, as usual, $T \mid \alpha=\bigcup\left\{T_{\beta}: \beta<\alpha\right\}$. We do this so that $T \mid \alpha$ is a $\lambda$-normal tree (each node not on the top level has $\lambda$ immediate successors), and for $\beta<\alpha, T_{\beta} \subseteq[\lambda \beta, \lambda(\beta+1))$ (ordinal multiplication, ordinal interval). We shall also have that for $i<\alpha, \bar{x}^{i}=\left(x_{n}^{i}: n<\omega\right)$ is a one-to-one sequence from $T_{i}$. The $\bar{x}^{i}$ form what Devlin and Laver called an ascent path [4], viz (1) below, showing that the tree $T=\bigcup\left\{T \mid \alpha: \alpha<\lambda^{+}\right\}$is not special. The function $f=\bigcup\left\{f \mid \alpha: \alpha<\lambda^{+}\right\}$ will press down on levels in $S$, showing that $T$ is a $\lambda^{+}-A$-tree. In the terminology of [6, Chapter IX], $T$ is $\lambda^{+}-S$-st-special.

The construction will have the following properties, which we take as induction hypotheses.

$$
i<j \Rightarrow x_{n}^{i} \triangleleft x_{n}^{j} \wedge\left\{f(z): z \in\left(x_{n}^{i}, x_{n}^{j}\right]_{\triangleleft}\right\} \cap \lambda i=\varnothing
$$

for sufficiently large $n$ (we use $(,]_{\triangleleft}$ and corresponding notation for $\triangleleft$-intervals, here and below), and if $i \in C_{j}^{\prime}$, then this holds for all $n$; the last assertion will, in fact, follow from (8) below, for $j \notin S$, but for perspicacity, we state it here. Further, looking ahead, note that the first assertion says that $x_{n}^{j}$ is the $w$ of (5) for $x_{n}^{i}$ and sufficiently large $n$.

(2) For $x \in T_{i}(i \in S \Rightarrow f(x)<\lambda i) \wedge(i \notin S \Rightarrow f(x)=x)$.

(3) $x \triangleleft y \Rightarrow f(x) \neq f(y)$ (we say $f$ is $1-1$ on chains).

(4) $\left(i<j \wedge y \in T_{j}\right) \Rightarrow \operatorname{card}\left(\{f(x): x \triangleleft y\} \cap T_{i}\right)<\lambda$.

(5) $\left(i<j \wedge y \in T_{i}\right) \Rightarrow\left(\exists w \in T_{j}\right) y \triangleleft w \wedge\left\{f(z): z \in(y, w]_{\triangleleft}\right\} \cap \lambda i=\varnothing$.

We shall also build a function $h$, defined on pairs of the form $(x, j)$ where $j \notin S$, $j$ is a positive limit ordinal, and $x \in T_{i}$ with $i \in C_{j}^{\prime}$. When this occurs, we will 
have $h(x, j) \in T_{j}$ and $x \triangleleft h(x, j)$. The following properties of $h$ will be additional induction hypotheses. The crucial (7) is similar to (5), but in the more general setting of (5), coherence as in (6) is not required (though it could be) so we do not single out the witness.

(6) $\left(i_{1}<i_{2}\right.$, both in $C_{j}^{\prime}$ with $\left.j \notin S \wedge x \in T_{i_{1}}\right) \Rightarrow h(x, j)=h\left(h\left(x, i_{2}\right), j\right)$.

(7) $\left(i \in C_{j}^{\prime} \wedge j \notin S \wedge x \in T_{i}\right) \Rightarrow\left\{f(z): z \in(x, h(x, j)]_{\triangleleft}\right\} \cap \lambda i=\varnothing$.

(8) $\left(i \in C_{j}^{\prime} \wedge j \notin S\right) \Rightarrow h\left(x_{n}^{i}, j\right)=x_{n}^{j}$ for all $n<\omega$.

$T_{0}=\lambda$. If $\alpha>0$, having constructed $T \mid \alpha$ with (1)-(8), we take $T_{\alpha}=[\lambda \alpha$, $\lambda(\alpha+1))$ and we assign an $\alpha$-branch of predecessors through $T \mid \alpha$ to each member of $T_{\alpha}$. Actually, as usual, what we do is to choose $\lambda \alpha$-branches through $T \mid \alpha$ to which we give tops; these are then identified with $[\lambda \alpha, \lambda(\alpha+1))$. If $\alpha$ is a successor ordinal, instead of branches we consider elements of $T_{\alpha-1}$; each of these gets $\lambda$ immediate successors, making sure that the point chosen to be $x_{n}^{\alpha}$ is among the successors of $x_{n}^{\alpha-1}$. Note that (4) and (5) are preserved; the preservation of (5) uses (5) for $\alpha-1$. This completes the successor case, so suppose $\alpha$ is a positive limit ordinal. Here we really do choose branches, putting one top on each chosen branch.

We consider three cases.

Case 1. $\alpha \notin S, C_{\alpha}^{\prime}$ is not cofinal in $\alpha$.

Case 2. $\alpha \notin S, C_{\alpha}^{\prime}$ is cofinal in $\alpha$.

Case 3. $\alpha \in S$ (so cf $\alpha=\omega$ ).

In Cases 1 and 2, we will, of course, set $f(x)=x$ for $x \in T_{\alpha}$. In Case 1, cf $\alpha=\omega$. In this case, if $C_{\alpha}^{\prime}=\varnothing$, set $\alpha_{0}=0$; otherwise, set $\alpha_{0}=\max C_{\alpha}^{\prime}$. Then choose $\left(\alpha_{n}: 0<n<\omega\right)$ strictly increasing and cofinal in $\alpha$ with $\alpha_{0}<\alpha_{1}$. For each $n<\omega$, let $p(n, n+1)<\omega$ be such that if $p(n, n+1) \leq m$ then $x_{m}^{\alpha_{n}} \triangleleft x_{m}^{\alpha_{n+1}}$ and $\{f(z): z \in$ $\left.\left(x_{m}^{\alpha_{n}}, x_{m}^{\alpha_{n+1}}\right]_{\triangleleft}\right\} \cap \lambda \alpha_{n}=\varnothing$. Let $q(0)=0, q(n+1)=\max \{q(n)+1, p(n, n+1)\}$. Thus, $(q(n) \leq m, l<n) \Rightarrow x_{m}^{\alpha_{l}} \triangleleft x_{m}^{\alpha_{n}}$. We first choose $\omega$ branches, whose tops will be the $x_{m}^{\alpha}$. We guarantee that, given $m$, letting $n$ be such that $q(n) \leq m<q(n+1)$, the $m$ th branch contains all the $x_{m}^{\alpha_{l}}$ for $l \leq n$. Above level $\alpha_{n}$, the $m$ th branch is generated by using (5) successively to obtain successors $y_{k+1}$ on level $\alpha_{n+k}$ for $k<\omega$; conventionally, we set $y_{0}=x_{m}^{\alpha_{n}}$. Then, $y_{k+1}$ will be the $w$ guaranteed by (5) for $i=\left|y_{k}\right|, j=\alpha_{n+k+1}, y=y_{k}$.

Now, for each $n$, and each $x \in T_{\alpha_{n}}$, we choose $\left(y_{k}: n \leq k<\omega\right)=\left(y_{k}^{x}: n \leq k<\right.$ $\omega)$, strictly $\varangle$ increasing, with $y_{n}=x, y_{k}$ on level $\alpha_{k}$. Again this uses (5), taking $y_{k+1}$ to be $w$, where $i=\alpha_{k}, j=\alpha_{k+1}, y=y_{k}$. If $C_{\alpha}^{\prime}=\varnothing$, there are no $h(x, \alpha)$ to define. Otherwise we define $h(x, \alpha)$ for $x$ on level $\alpha_{0}$; the other $h(x, \alpha)$ will be defined in terms of these and the $h\left(x, \alpha_{0}\right)$ to guarantee commutativity, property (6). We simply take $h(x, \alpha)$ to be the top of the branch generated by the $\left(y_{k}: k<\omega\right)$ for $x$ on level $\alpha_{0}, x \notin\left\{x_{m}^{\alpha_{0}}: m<\omega\right\}$. We set $h\left(x_{m}^{\alpha_{0}}, \alpha\right)=$ the top of the $m$ th special branch $\left(=x_{m}^{c}\right)$. Then, properties (6)-(8) are clear by construction.

Property (2) is preserved, vacuously, since $\alpha \notin S$. Property (3) is preserved, since if $y \in T_{\alpha}$, then, since $\alpha \notin S, f(y)=y$. Property (4) is clear, since every branch extended at level $\alpha$ was generated using (5) and, therefore we have

$$
(\forall i<\alpha)\left(\forall y \in T_{\alpha}\right)(\exists z \triangleleft y)\{f(x): x \triangleleft y\} \cap T_{i}=\{f(x): x \triangleleft z\} \cap T_{i} .
$$

Applying the induction hypothesis (4) to such a $z$, the conclusion is clear. Property (5) is clear when $i=\alpha_{n}, j=\alpha$, simply taking $w=$ the top of the branch $\left(y_{k}: n \leq\right.$ $k<\omega$ ); if $i<\alpha_{n}$, using (5) when $j=\alpha_{n}$ (which is an induction hypothesis) and the 
preceding case, the conclusion is clear. Finally, (1) is clear by construction when $i=\alpha_{n}, j=\alpha$. For $i<\alpha_{n}$, we use (1) as an induction hypothesis with $j=\alpha_{n}$ and the preceding case, as with (5).

In Case 2, we have the natural branches $b_{x}=$ the branch generated by $\{x\} \cup$ $\left\{h(x, \gamma):|x|<\gamma \in C_{\alpha}^{\prime}\right\}$, for $|x| \in C_{\alpha}^{\prime}$. This is, in fact, a branch by the coherence property of the $\square$-sequence and the coherence property (6), of the $h(x, \gamma)$ 's. Of course, $h(x, \alpha)=$ the top of this branch. These are the only branches which receive tops. Note that, by the induction hypothesis (8), for $\beta \in C_{\alpha}^{\prime}, n<\omega, x=x_{n}^{\beta}, b_{x}=$ the branch generated by $\{x\} \cup\left\{x_{n}^{\gamma}: \beta<\gamma \in C_{\alpha}^{\prime}\right\}$, so we take $x_{n}^{\alpha}=$ the top of this $b_{x}$ (the choice of $\beta$ being immaterial). All properties except (4) are now clear by construction ((1) and (5) require arguments as in Case 1 , when $\left.i \notin C_{\alpha}^{\prime}\right)$. For (4), we use the induction hypothesis (7), the construction of the $b_{x}$ 's and the fact that these are the only branches given tops. As in Case 1, this guarantees that if $i \leq \beta \in C_{\alpha}^{\prime}$, $y \in T_{\alpha},\{f(x): x \triangleleft y\} \cap T_{i}=\{f(x): x \triangleleft y$ and $|x|<\beta\} \cap T_{i}=\left\{f(x): x \triangleleft y^{\prime}\right\} \cap T_{i}$, where $y^{\prime}$ is the predecessor of $y$ on level $\beta$, and we apply the induction hypothesis (4) with $y=y^{\prime}, j=\beta$.

In Case 3, cf $\alpha=\omega$ and our method of constructing branches to extend to level $\alpha$ parallels that of Case 1, even though we may have $C_{\alpha}^{\prime}$ cofinal in $\alpha$. Note that, here, we are not required to define the $h(x, \alpha)$ nor to preserve (6)-(8). Here, however, we must make $f$ press down and preserve (3)-(5). So, we set $\alpha_{0}=0$, and proceed essentially as in Case 1, with some refinements designed to handle (5). First, we choose branches to be topped by the $x_{m}^{\alpha}$, as in Case 1, also guaranteeing that if $q(n) \leq m$ then $\lambda \alpha_{n} \leq f\left(x_{m}^{\alpha}\right)$.

The remaining branches which are extended will each arise from $\omega$ applications of (5), as in Case 1. Now, each such branch starts from a pair $(i, y)$ where $i<\alpha$ and $y \in T_{i}$. The set of all such pairs gives the set of instances of (5) which must be handled. We enumerate all such pairs in order type $\lambda$ and use that at any stage in the construction of the branches, fewer than $\lambda$ points have been used. We then proceed by choosing $y^{\prime}$, an immediate successor of $y$ which does not occur in any of the previously chosen branches, and repeatedly applying (5) to the $\alpha_{n}$ which lie above $\left|y^{\prime}\right|$, starting from $y^{\prime}$, as in Case 1 . Note that $f\left(y^{\prime}\right)=y$, by (2), and that distinct pairs $(i, y)$ give rise to distinct $y^{\prime}$ and therefore to distinct branches. Then, if $w$ has been chosen as the top of the $(i, y)$ branch, we let $f(w)$ be any member of $T_{|y|}$, distinct from $y$ and all the $f\left(w^{\prime}\right)$, where $w^{\prime}$ is the top of a previously chosen branch. Now (3) and (5) are clear by construction, and (4) is not problematical, arguing as in Case 1. This completes the construction. Of course, since $T$ is $\left(\lambda^{+}-S\right.$-st-special $)$, it is a $\lambda^{+}-A$-tree.

2. Lemma 2. Throughout this section we assume that $\mathrm{cf} \lambda=\omega$ and that $\left(C_{\delta}: 0<\delta=\bigcup \delta<\lambda^{+}\right)$is a $\square_{\lambda}$-sequence, $S \subseteq\left\{\delta<\lambda^{+}: \operatorname{cf} \delta=\aleph_{1}\right\}, S$ is stationary but for all $\delta, C_{\delta}^{\prime} \cap S=\varnothing$.

Again, we construct $T \mid \alpha, f,\left(x_{\xi}^{i}: \xi<\omega_{1}\right)$, essentially as in $\S 1$, with the same induction hypotheses, but, of course, with $\omega_{1}$ replacing $\omega$ as the width of the ascent paths. The division into cases is also the same, as is the construction in Case 2 of $\S 1$. The construction in Case 1 of $\S 1$ is, if anything, simpler, since we can let $\xi_{0}$ be such that whenever $l<n$ and $\xi_{0} \leq \xi, x_{\xi}^{\alpha_{l}} \triangleleft x_{\xi}^{\alpha_{n}}$. Then, for $\xi_{0} \leq \xi$, we let the $\xi$ th branch be the branch generated by $\left\{x_{\xi}^{\alpha_{n}}: n<\omega\right\}$. For $\xi<\xi_{0}$, we choose the $\xi$ th branch to avoid all the $x_{\xi}^{\alpha_{n}}$ for $n<\omega$. 
There remains Case 3 of $\S 1$. Here, $\operatorname{cf} \alpha=\omega_{1}$, so $C_{\alpha}^{\prime}$ is cofinal in $\alpha$. As in Case 2, if $i \in C_{\alpha}^{\prime}$ and $x \in T_{i}$, then $\left\{h(x, k): k \in C_{\alpha}^{\prime} \backslash(i+1)\right\}$ generates a branch $b_{x}$. Some of these branches will be extended.

First choose the branches to be topped with the $x_{\xi}^{\alpha}$ as in Case 2. Since cf $\alpha=\omega_{1}$, we define the $f\left(x_{\xi}^{\alpha}\right)$ so that

$$
(\forall i<\alpha)\left(\exists \xi_{0}<\omega_{1}\right)\left(\forall \xi \in\left(\xi_{0}, \omega_{1}\right)\right) \lambda i \leq f\left(x_{\xi}^{\alpha}\right) .
$$

This guarantees (1). Then, as before, list all pairs $(i, y)$ with $i<\alpha$ and $y \in T_{i}$ in type $\lambda$ and handle each pair in turn by choosing $y^{\prime}$ an immediate successor of $y$ not appearing in any branch chosen so far and choose $k \in C_{\alpha}^{\prime} \backslash(i+2)$ and a successor of $y^{\prime}$ on $T_{k}$ with $\left\{f(z): z \in\left(y^{\prime}, x\right]_{\triangleleft}\right\} \cap \lambda(i+1)=\varnothing$, by (5). Then, top $b_{x}$ with a point $w$ at level $\alpha$ and require that $\lambda i \leq f(w)$. For each of the remaining induction hypotheses, the verification that it is preserved is either clear from the construction or totally analogous to the verification in $\S 1$.

3. Lemma 3 and Theorem 4. We first note that Theorem 4 is an immediate Corollary of Lemmas $1-3$, taking $\kappa$, in Lemma 3 , to be $\aleph_{0}$ or $\aleph_{1}$ according to whether $\operatorname{cf} \lambda>\aleph_{0}$ or $\operatorname{cf} \lambda=\aleph_{0}$.

So, suppose that $T, \varsigma(\alpha)\left(\alpha<\lambda^{+}\right)$, and $\left(x_{i}^{\alpha}: i<\kappa\right)$ are as in the statement of Lemma 3 and suppose, towards a contradiction, that $f: T \rightarrow \lambda$ is one-to-one on chains. For $\beta<\lambda^{+}, y \in T_{\beta}$, and $j<\beta$, let $\operatorname{pr}_{j}(y)=$ the predecessor of $y$ on $T_{j}$. Let $\theta<\lambda$ and let $S_{\theta}=\left\{\delta<\lambda^{+}: \operatorname{cf} \delta>\kappa, \theta\right\}$, so $S_{\theta}$ is stationary. Let $\delta \in S_{\theta}$. Note that for each $i<\kappa,\left\{\alpha<\delta: f\left(\operatorname{pr}_{\alpha}\left(x_{i}^{\delta}\right)\right)<\theta\right\}$ has power at most $\operatorname{card} \theta$, and therefore is bounded in $\delta$, say with sup $=h_{i}(\delta)$. Since cf $\delta>\kappa$, $h(\delta)=_{\text {def }} \sup \left\{h_{i}(\delta)+1: i<\kappa\right\}<\delta$. Now, $h$ is constant on a stationary subset $S_{\theta}^{*}$ of $S_{\theta}$, say with value $\alpha_{\theta}$. Suppose, now, that $\alpha_{\theta}<\beta<\lambda^{+}$. Let $\delta \in S_{\theta}^{*} \backslash(\varsigma(\beta)+1)$. Then, for some $i<\kappa, i \leq j \Rightarrow x_{j}^{\beta} \triangleleft x_{j}^{\delta}$. We let $i(\beta, \delta)=$ the least such $i$. Thus, for $i(\beta, \delta) \leq j<\kappa, x_{j}^{\beta}=\operatorname{pr}_{s(\beta)}\left(x_{j}^{\delta}\right)$, and therefore, since $h_{j}(\delta)<\alpha_{\theta}<\beta \leq \varsigma(\beta)$, $\theta \leq f\left(x_{j}^{\beta}\right)$. Summing up, we have shown

$$
\alpha_{\theta}<\beta<\lambda^{+} \Rightarrow \theta \leq f\left(x_{j}^{\beta}\right) \quad \text { for large enough } j<\kappa
$$

Let $\alpha^{*}=\sup \left\{\alpha_{\theta}: \theta<\lambda\right\}$, so $\alpha^{*}<\lambda^{+}$. But now we get a contradiction, as follows. First, choose $\beta \in\left(\alpha^{*}, \lambda^{+}\right)$; then for all $\theta<\lambda, \theta \leq f\left(x_{j}^{\beta}\right)$ for sufficiently large $j$. Let $i(\theta)$ be least so that for $i \leq j, \theta \leq f\left(x_{j}^{\beta}\right)$. Clearly $i$ is a nondecreasing function of $\theta$ and since range $f \subseteq \lambda, i: \lambda \rightarrow \kappa$ is cofinal, contradicting $\lambda \neq \kappa=\operatorname{cf} \kappa$.

REMARKS. In early versions of this paper, in place of Lemma 3, we had (incorrectly stated) hypotheses on cardinal exponentiation which (had they been correctly stated) permitted an easier but far less informative proof that the trees constructed in $\S \S 1$ and 2 are special. Later, Shelah came up with the proof of Lemma 3 in the special case cf $\lambda>\kappa$, which he showed to S. Ben David and S. Todorcević. Todorcević suggested a simplification, whereupon Shelah proved the theorem in general.

4. Theorem 5. We assume, without loss of generality, that $\lambda$ is regular, since if not, then either $\square_{\lambda}$ holds, in which case there is a nonspecial $\lambda^{+}-A$-tree, or the 
Covering Lemma (for $K$, but even the Covering Lemma for $L$ is sufficient for our purposes) fails and so, clearly, $\kappa$ is (weakly compact) ${ }^{L}$. We will use the following lemma to complete the proof of Theorem 5 .

LEMMA 6. If $\lambda$ is regular and uncountable and $\lambda^{+}$is not (weakly compact) ${ }^{L}$, then either $\square_{\lambda}$ or there is a nonspecial $\lambda^{+}-A$-tree.

REMARKS. The form in which we have stated Lemma 6 may seem rather bizarre, since, in view of Theorem 4 , there is no need for the dichotomy, but we have stated it in this form in order to make clearer several historical points. First, we should note that in our early work on this problem, we had proved a somewhat weaker form of this lemma with the same dichotomy but with a nonspecial $\lambda^{+}$wide tree in place of a nonspecial $\lambda^{+}-A$-tree. Todorcević then pointed out that his techniques, from [11], yielded the stronger conclusion, but also that, while Lemma 6 is not explicitly stated in [11], most of the ingredients for proving it do appear there.

Further historical remarks will appear at various places in the proof of Lemma 6, but before turning to the proof, let us just note that by Theorem 4 and the above reduction to regular $\lambda$, Theorem 5 is immediate from Lemma 6 .

PROOF OF LEMMA 6 . We first give an outline of how the proof will proceed. We let $\kappa=\lambda^{+}$. We follow Todorcević by calling a sequence $\left(C_{\alpha}: \alpha\right.$ a limit ordinal $<\mu$ ) of clubs of $\alpha$, with the coherence property of a $\square$-sequence and which is not trivialized by a club of $\mu$ (i.e. there is no club $C \subseteq \mu$ such that for all $\alpha \in C$, $\left.C_{\alpha}=C \cap \alpha\right)$, a $\square(\mu)$-sequence. Note that we have not required the order types of the $C_{\alpha}$ 's to be bounded: this would essentially give $\square$ (really, if, as below, $\mu=\kappa$ and the bound is $\lambda$ ). Below, we shall connect this with the question of whether a certain tree is special.

The first step in the proof of Lemma 6 is to prove Proposition 6.1 below; a slightly stronger statement is widely attributed (e.g. in $[\mathbf{1 1}, \mathbf{1 2}])$ to Jensen, unpublished. In view of the fact that the proof of Theorem 6.1 of [3] contains all but one of the important ingredients of a proof of Proposition 6.1, this attribution is no doubt justified. We shall give a "gloss" of Jensen's proof and provide the missing ingredient, a well-known well-foundedness of direct limit argument. The "proof" (of the stronger statement) given in [12] is incorrect, probably as a result of oversimplification. Arguments and constructions similar to those of the proof of Proposition 6.1 appear in [5]; our approach can be used to give an alternate proof of a stronger form of Magidor's theorem, there, that if a regular $\mu$ is not (weakly compact) ${ }^{L}$, then there are stationary subsets $S_{1}, S_{2}$ of $\mu$ such that for no $\alpha<\mu$ do we have both $S_{1} \cap \alpha$ and $S_{2} \cap \alpha$ stationary in $\alpha$. Our strengthening gets such $S_{1}$, $S_{2}$ to both be constructible; see the closing remarks below.

PROPOSITION 6.1. If $\mu>\omega_{1}$ is a cardinal which is (regular) ${ }^{L}$ but not (weakly compact $)^{L}$ and $\mathrm{cf} \mu>\omega$, then there is a constructible sequence $\left(\bar{C}_{\alpha}: \alpha\right.$ a limit ordinal $<\mu$ ) which is a $\square(\mu)$-sequence (in $V$ ).

Before proving the proposition, we indicate how we will finish the proof of Lemma 6. Let $\kappa=\lambda^{+}=\mu$, and let $\bar{C}=\left(\bar{C}_{\alpha}: \alpha\right.$ a limit ordinal $\left.<\kappa\right)$ be as guaranteed by the proposition. The natural tree based on $\bar{C}$ has the limit ordinals $<\kappa$ as node set and has $\alpha$ precede $\beta$ if $\alpha<\beta$ and $\alpha \in \bar{C}_{\beta}^{\prime}$. Note that the fact that $\bar{C}$ is not trivialized by a club of $\kappa$ is equivalent to the nonexistence of $\kappa$-branches 
in the natural tree. We shall then show that either the natural tree based on $\bar{C}$ is nonspecial (unfortunately, it may be wide; this was as far as we had gotten in our original proof, mentioned above after the statement of Lemma 6) or else real $\square_{\lambda}$, in which case we invoke Lemma 1 (here is the connection with the notion of specialness mentioned above; note that if $\bar{C}$ is a $\square_{\lambda}$-sequence, the natural tree is clearly special; what we will prove right after the proof of Proposition 6.1, below, is not an exact converse, but almost). Finally, in the first case Todorcević suggested embedding the natural tree based on $\bar{C}$ into the $\rho_{0}$-tree, where $\rho_{0}$ is based on $\bar{C}$ (see [11] for this terminology and other material concerning $\rho_{0}$ and related notions). We shall then invoke arguments from [11] which show that, in our context, the $\rho_{0}$-tree is not wide and we conclude by the easy observation that it is not special, lest the natural tree based on $\bar{C}$ be special.

PROOF OF PROPOSITION 6.1. First note that since $\mu$ is an uncountable cardinal which is not (weakly compact) ${ }^{L}, 0^{\sharp}$ does not exist, so the Covering Lemma for $L$ holds, so, since $\mu$ is a cardinal which is (regular) ${ }^{L}$, in fact $\mu$ is regular. Now the setting of Theorem 6.1 of [3] is as follows: $\kappa$ is a cardinal which is $>\left(\omega_{1}\right)^{L}$ and which, in $L$, is regular, but not weakly compact. The theorem asserts the existence of a constructible, constructibly stationary, $E \subseteq \kappa$ which is avoided by all members of a sequence $\bar{C}=\left(\bar{C}_{\lambda}: \lambda\right.$ limit, $\left.\lambda<\kappa\right)$, with $\bar{C}_{\lambda}$ club in $\lambda$. This, of course, guarantees that $E$ is nonreflecting, which is a strong form of evidence that the sequence of clubs is a $\square(\kappa)$-sequence, at least in $L$. In fact, we will show that this sequence is a $\square(\kappa)$-sequence in $V$, but the nature of the evidence for this will not be as strong as the existence of a nonreflecting stationary set ( $E$, of course, will continue to have its initial segments nonstationary; unfortunately, we do not see how to prove that $E$ itself remains stationary in $V$ ).

To harmonize with the notation of Proposition 6.1, in the preceding paragraph, everywhere replace $\kappa$ by $\mu$ and $\lambda$ by $\alpha$. Also assume that in $V, \operatorname{cf} \mu>\omega$. Further, while the next manuever is not really necessary, it is built into Jensen's construction and will somewhat facilitate matters: we assume that $\mu$ is (inaccessible) ${ }^{L}$. We argue that this assumption is benign, for our purposes, since if $\mu$ is not (inaccessible) ${ }^{L}$, then it is a successor, in $L$, say of $\mu_{0}$. But then $\square_{\mu_{0}}$ holds in $L$ and we claim that a constructible $\square_{\mu_{0}}$-sequence is a $\square(\mu)$-sequence in $V$. This is clear, since $\mu$ is regular in $V$ and since the order types of the $C_{\alpha}$ in the $\square_{\mu_{0}}$-sequence are bounded by $\mu_{0}$.

The impact of our assumption that $\mu$ is (inaccessible) ${ }^{L}$ is that $\Lambda \underset{\text { def }}{=}\{\eta<\mu$ : ( $\eta$ is a limit cardinal $\left.)^{L}\right\}$ is club in $\mu$. Since $\mu$ is not (weakly compact) ${ }^{L}$, we fix a constructible counterexample to $\Pi_{1}^{1}$-indescribability, i.e. a $B \in \mathscr{P}(\mu) \cap L$ such that for some first order $\theta$ in $\in$ and two additional unary predicate symbols, $\theta$ holds in $\left(J_{\mu}, \in, B, D\right)$ for all $D \in \mathscr{P}(\mu) \cap L$, but for all $\alpha<\mu$ there is some $D \in \mathscr{P}(\alpha) \cap L$ such that $\theta$ fails in $\left(J_{\alpha}, \in, B \cap \alpha, D\right)$.

Now let $\alpha<\mu$ be a limit ordinal. The first division into cases is whether or not $\alpha \in \Lambda$. If not, then certainly $\Lambda$ is bounded in $\alpha$, so let $\alpha_{0}=\max ((\Lambda \cup\{0\}) \cap \alpha)$, and let $\bar{C}_{\alpha}=\alpha \backslash \alpha_{0}$. Note that

(1) Clearly there can be no stationary $S \subseteq \mu \backslash \Lambda$ such that for $\alpha<\beta$, both in $S$, $\alpha \in \bar{C}_{\beta}^{\prime}$, since $\Lambda$ is club in $\mu$.

Jensen next defines two sets $Q \subseteq Q^{\prime} \subseteq \Lambda$. We discuss below the definition of $\bar{C}_{\alpha}$ for $\alpha \in Q$. $Q$ will contain all the (inaccessible cardinals) ${ }^{L}$ below $\mu$. There are four cases for $\alpha \in \Lambda \backslash Q$. Since these $\alpha$ are (singular) ${ }^{L}$, the global $\square$-sequence of 
Theorem 5.1 of [3] already assigns to $\alpha$ a club $C_{\alpha}$. Let $\tilde{C}_{\alpha}$ be $\Lambda \cap C_{\alpha}^{\prime}$, i.e. the set of (limit cardinals) ${ }^{L}$ below $\alpha$ which are limit points of $C_{\alpha}$. For $\alpha \in \Lambda \backslash Q$, Case 1 of the definition of $\bar{C}_{\alpha}$ is: $\bar{C}_{\alpha}$ is bounded in $\alpha$. Then, $\operatorname{cf}^{L} \alpha=\omega$, and $\bar{C}_{\alpha}$ is taken to be any constructible, cofinal subset of $\alpha$ of order type $\omega$. It is clear that

(2) There is no stationary $S \subseteq \mu$ of these "Case 1 " ordinals such that for $\alpha<\beta$, both in $S, \alpha \in \bar{C}_{\beta}^{\prime}$.

In dealing with Cases 2-4 of the definition of $\bar{C}_{\alpha}$, for $\alpha \in \Lambda \backslash Q, \bar{C}_{\alpha}$ is defined as a subset of $\tilde{C}_{\alpha}$, which, in turn, is a subset of $C_{\alpha}$, which will have order type $<\alpha$; we shall explain why momentarily, by appealing to the construction of $C_{\alpha}$. This also has four cases, and we shall first present these and give the correspondence between the cases of the definition of $\bar{C}_{\alpha}, \alpha \in \Lambda \backslash Q$, and those of the definition of $C_{\alpha}$.

The first two cases in the definition of $C_{\alpha}$ involve ordinals not in $\Lambda$, so they do not arise in discussion of the four cases of the definition of $\bar{C}_{\alpha}$ for $\alpha \in \Lambda \backslash Q$. For $\alpha \in \Lambda$, Case 3 in the definition of $C_{\alpha}$ is subsumed by Case 1 , above, of the definition of $\bar{C}_{\alpha}$ for $\alpha \in \Lambda \backslash Q$. It is worth noting that the ordinals in Jensen's $E$ as well as those in $Q^{\prime} \backslash Q$ all fall under this case.

Cases 2-4 of the definition of $\bar{C}_{\alpha}$, for $\alpha \in \Lambda \backslash Q$, fall under Case 4 of the definition of $C_{\alpha}$. As we have already noted above, in Cases $2-4, \bar{C}_{\alpha}$ is defined as a subset of $\tilde{C}_{\alpha}$, which, in turn is a subset of $C_{\alpha}$. According to the construction of $C_{\alpha}$ in Case $4, C_{\alpha}$ has order type $<\alpha$ : the relevant facts are that the functions $k, l, m$ of Theorem 5.1 of [3] are defined on a limit ordinal $\theta<\gamma<\alpha$ (p. 276 of [3], following the proof of Lemma 5.4) and that $C_{\alpha}$ is $l^{\prime \prime} t^{\prime \prime} \tilde{\theta}$, where $t: \tilde{\theta} \rightarrow \theta$ is normal so that $\tilde{\theta} \leq \theta[3$, p. 279]. We now argue that

(3) There is no stationary subset $S$ of "cases $2-4$ " limit ordinals from $\Lambda \backslash Q$ such that for all $\alpha<\eta, \alpha, \eta \in S, \alpha \in \bar{C}_{\eta}^{\prime}$.

Suppose, towards a contradiction there were such an $S$. By Fodor's lemma (recall that $\mu$ is regular, in $V$ ) there is stationary $S^{\prime} \subseteq S$ on which o.t. $\bar{C}_{\alpha}$ is constant. This contradicts the assumed property of $S$.

Finally, we deal with the set $Q$. Jensen defined

(*) $\alpha \in Q^{\prime}$ iff there is a $\beta>\alpha$ such that $\alpha$ is (regular) $)^{J_{\beta+1}}, B \cap \alpha \in J_{\beta}$ and there is a $D \in \mathscr{P}(\alpha) \cap J_{\beta}$ such that $\theta$ fails in $\left(J_{\alpha}, \in, B \cap \alpha, D\right)$.

If $\alpha \in Q^{\prime}$ and $\beta$ is the least witness, then $\alpha \in Q$ iff whenever $p \in J_{\beta}$, there is an elementary substructure $A$ of $J_{\beta}$, containing $p$, whose intersection with $\alpha$ is an ordinal $<\alpha$. To define $\bar{C}_{\alpha}$ for $\alpha \in Q$, let $\beta$ be the least witness to $\alpha \in Q^{\prime}$. We form the usual tower of elementary substructures of $J_{\beta}$, containing $\alpha$ and $B \cap \alpha$ and with transitive intersections with $\alpha$ (note that if $d(\alpha)$ is the $<_{J_{\beta}}$-least $d \subseteq \alpha$ such that $\theta$ fails in $\left(J_{\beta}, \in, B \cap \alpha, d\right)$, then $d(\alpha)$ is in any such substructure, by elementarity). Then, $\bar{C}_{\alpha}$ is the set of intersections with $\alpha$ of all such substructures. Jensen goes on to show [3, Lemma 6.4, p. 289] that if $\alpha^{\prime} \in \bar{C}_{\alpha}^{\prime}$, then $\alpha^{\prime} \in Q$ and $\bar{C}_{\alpha^{\prime}}=\bar{C}_{\alpha} \cap \alpha^{\prime}$.

He does so by showing that if $\beta^{\prime}$ is the ordinal of the transitivization of the elementary substructure whose intersection with $\alpha$ is $\alpha^{\prime}$, then $\beta^{\prime}$ is the least witness to the fact that $\alpha^{\prime} \in Q^{\prime}$. In other notation, if, for $\alpha \in Q$, we let $\beta(\alpha)$ be the least witness to the fact that $\alpha \in Q^{\prime}$ and we let $\mathscr{A}(\alpha)=\left(J_{\beta(\alpha)}, \in, B \cap \alpha, \alpha\right)$, we have that if $\alpha^{\prime} \in \bar{C}_{\alpha}^{\prime}$, then there is $\pi_{\alpha^{\prime} \alpha}$, an elementary embedding of $\mathscr{A}\left(\alpha^{\prime}\right)$ into $\mathscr{A}(\alpha)$ 
which is the identity on $\alpha^{\prime}$, maps $\alpha^{\prime}$ to $\alpha$, and $B \cap \alpha^{\prime}$ to $B \cap \alpha$. Further, we clearly have $\pi_{\alpha^{\prime} \alpha}\left(d\left(\alpha^{\prime}\right)\right)=d(\alpha)$. Finally, if $\alpha \in \bar{C}_{\eta}^{\prime}$, then $\pi_{\alpha^{\prime} \eta}=\pi_{\alpha \eta} \circ \pi_{\alpha^{\prime} \alpha}$.

Now, if there were a cofinal $X \subseteq \mu \cap Q$ such that for $\alpha^{\prime}<\alpha$, both in $X, \alpha^{\prime} \in \bar{C}_{\alpha}^{\prime}$, we could take the direct limit of the commutative system $\left(\left(\mathscr{A}(\alpha), \pi_{\alpha^{\prime} \alpha}: \alpha^{\prime}<\alpha\right.\right.$, both in $X)$. This would clearly be well-founded, extensional, rudimentarily closed and a model of the $\Pi_{2}$ sentence "I am a $J_{\beta}$ ", so we could take its underlying set to be $J_{\beta^{\prime}}$, for some $\beta$. Calling the direct limit $\left(\left(\mathscr{A}, \pi_{\alpha^{\prime}}\right): \alpha^{\prime} \in X\right)$, it is easy to see that $\pi_{\alpha^{\prime}}\left(\alpha^{\prime}\right)=\mu$ (so $\left.\beta>\mu\right), \pi_{\alpha^{\prime}}\left(B \cap \alpha^{\prime}\right)=B$, and that, letting $D$ be the common value of the $\pi_{\alpha^{\prime}}\left(d\left(\alpha^{\prime}\right)\right)$, we must have $\theta$ fails in $\left(J_{\mu}, \in, B, D\right)$, which is impossible. Thus,

(4) There is no such $X$.

But then $\left(\bar{C}_{\alpha}: \alpha\right.$ is a limit ordinal $\left.<\mu\right)$ is a $\square(\mu)$-sequence in $V$ by (1)-(4). This completes the proof of Proposition 6.1.

We take up the thread of the proof of Lemma 6, so $\kappa=\lambda^{+}$(= the $\mu$ of Proposition $6.1)$, and $\lambda$ is regular. Assume that $(T, \triangleleft)$ is the natural tree based on $\bar{C}$ and that $f: T \rightarrow \lambda$ is a specializing function. For limit $\delta<\kappa$, we define strictly increasing continuous $\left(\gamma_{i}: i<i(\delta)\right)=\left(\gamma_{i}^{\delta}: i<i(\delta)\right)$ from $C_{\delta}$, cofinal in $\delta$, where $i(\delta) \leq \lambda$ and we set $C_{\delta}^{*}=\left\{\gamma_{i}: i<i(\delta)\right\}$. If $C_{\delta}^{\prime}$ is not cofinal in $\delta$, let $\left(\gamma_{i}: i<\omega\right)$ be any increasing $\omega$-sequence cofinal in $\delta$, so suppose $C_{\delta}^{\prime}$ is cofinal in $\delta$. Conventionally, set $\gamma_{-1}=0$; thus the definition of $\gamma_{0}$ fails under the successor case. If $-1 \leq i$ and $\gamma_{i}$ has been defined, since $f$ is one-to-one on $C_{\delta}^{\prime}$, let $\gamma_{i+1}$ be the unique $\gamma \in$ $C_{\delta}^{\prime} \backslash\left(\gamma_{i}+1\right)$ such that $f(\gamma)=\inf f^{\prime \prime}\left(C^{\prime} \backslash\left(\gamma_{i}+1\right)\right)$. For limit $i, \gamma_{i}=\sup \left\{\gamma_{j}: j<i\right\}$. Let $i(\delta)$ be such that $\gamma_{i(\delta)}=\delta$. By the definition of $\gamma_{i+1}$, if $i+1<j$, then $f\left(\gamma_{i+1}\right)<f\left(\gamma_{j}\right)$, i.e. $f\left(\gamma_{i+1}: i<i(\delta)\right)$ is a strictly increasing sequence from $\lambda$. Thus $i(\delta) \leq \lambda$. We set $C_{\delta}^{*}=\left\{\gamma_{i}: i<i(\delta)\right\}$. The coherence property is easy to check. This completes the construction: $\left(C_{\delta}^{*}: \delta<\kappa, \delta\right.$ limit $)$ is a $\square_{\lambda}$-sequence.

We now assume that the natural tree based on $\bar{C}$ is not special. We first provide the relevant information from [11] about the function $\rho_{0}$ based on $\bar{C}$ (we take $\bar{C}$ to be defined on successor ordinals by setting $C_{\alpha}=\{\alpha\}$ ). Following [11], we let $\mathbf{Q}_{\kappa}$ be the finite sequences from $\kappa$, linearly ordered by the $\kappa$-analogue of the BrouwerKleene ordering. $\sigma \mathbf{Q}_{\kappa}$ is the tree of well-ordered chains of $\mathbf{Q}_{\kappa}$, ordered by initial segment.

(1) $\rho_{0}$ is a function from the graph of $\leq$ on $\kappa$ to $\mathbf{Q}_{\kappa}$.

(2) $\rho_{0}(\alpha, \alpha)=\varnothing ; \rho_{0}(\alpha, \beta)=\left\langle\text { o. t. }\left(C_{\beta} \cap \alpha\right)\right\rangle^{\wedge} \rho_{0}\left(\alpha, \min \left(C_{\beta} \backslash \alpha\right)\right)$.

(3) For all $\alpha, \rho_{0}(\xi, \alpha)$ is strictly increasing as a function of $\xi$; therefore, setting $T\left(\rho_{0}\right)=\left\{\rho_{0}(\cdot, \beta) \mid \alpha: \alpha \leq \beta<\kappa\right\}, T\left(\rho_{0}\right)$ is a subtree of $\sigma \mathbf{Q}_{\kappa}$.

(4) $\left.\operatorname{card}\left(T\left(\rho_{0}\right)_{\alpha}\right) \leq \operatorname{card}\left\{C_{\beta} \cap \alpha: \alpha \leq \beta<\kappa\right\}\right)+\aleph_{0}$.

(5) Since $\bar{C}$ is not trivialized by a club of $\kappa$, there is no $\kappa$-branch through $T\left(\rho_{0}\right)$.

(3) is clear from the definition (2). (4) is Lemma 1.2 on p. 4 of [11]; further, by the coherence property of $\bar{C}$, the RHS of the inequality of (4) is $\leq$ card $\alpha$. To see this, let $\alpha \leq \beta<\kappa$. Let $\gamma$ be the largest limit point of $C_{\beta} \cap \alpha$, if this last is infinite, or $\gamma=0$ if not. In either case, there are only card $\alpha$ possibilities for $\gamma$ and only card $\alpha$ possibilities for $C_{\beta} \cap[\gamma, \alpha)$, since this last is always finite. Finally, either $\gamma=0$ or $C_{\beta} \cap \alpha=C_{\gamma} \cup\left(C_{\beta} \cap[\gamma, \alpha)\right)$.

(5) is Lemma 1.6 of [11], together with the remark (ii) in $\S 1$ of [11], since $\bar{C}$ has the coherence property (property (i) in $\S 1$ of [11]). But (5), and the immediately preceding strengthening of (4) together yield that $T\left(\rho_{0}\right)$ is $\kappa$-Aronszajn. 
It remains to embed the natural tree based on $\bar{C}$ into $T\left(\rho_{0}\right)$ and to observe that this embedding preserves nonspecialness. The embedding is quite simple: $\pi(\beta)=\rho_{0}(\cdot, \beta)$; it is immediate from the definition of $\rho_{0}$ and the coherence property of $\bar{C}$ that if $\alpha \in\left(C_{\beta}\right)^{\prime}, \alpha<\beta$, then for all $\gamma<\alpha, \rho_{0}(\gamma, \alpha)=\rho_{0}(\gamma, \beta)$. Now if $f: T\left(\rho_{0}\right) \rightarrow \lambda$ is one-to-one on chains of $T\left(\rho_{0}\right)$, define $g:(\operatorname{Lim} \cap \kappa) \rightarrow \lambda$ by $g(\beta)=f(\pi(\beta))$. Clearly $g$ is also one-to-one on chains, since if $\alpha \in\left(C_{\beta}\right)^{\prime}$ and $g(\alpha)=g(\beta)$, then, as we have remarked, $\pi(\alpha) \subseteq \pi(\beta)$ and by definition of $g$, $f(\pi(\alpha))=g(\alpha)=g(\beta)=f(\pi(\beta))$, contradicting that $f$ is one-to-one on chains of $T\left(\pi_{0}\right)$. This completes the proof of Lemma 5 and Theorem 6 .

REMARKS. We provide the above-promised strengthening of Magidor's result from [5]. Let $\bar{C}$ be as in Proposition 6.1. Let $A_{\xi}^{0}=\left\{\alpha: \xi \notin \bar{C}_{\alpha}\right\}$ and $A_{\xi}^{1}=\{\alpha: \xi \in$ $\left.\bar{C}_{\alpha}\right\}$, so both are constructible. Clearly there is no $\xi, \beta$ such that both of the $A_{\xi}^{i} \cap \beta$ are stationary in $\beta$, so it will suffice to show that

(*) for some $\xi$, both $A_{\xi}^{i}$ are stationary.

If not, for $\xi<\kappa$, we can define $\varepsilon(\xi) \in 2$ by $\varepsilon(\xi)$ is that $\varepsilon \in 2$ such that $A_{\xi}^{\varepsilon}$ includes a club; also, let $D(\xi)$ be such a club. Let $C$ be the diagonal intersection of the $D(\xi)$. Now, if $\alpha<\beta$, both in $C^{\prime}$, then for $\xi<\alpha, \alpha, \beta \in D(\xi) \subseteq A_{\xi}^{\varepsilon(\xi)}$, so $\xi \in C_{\alpha}$ iff $\alpha \in A_{\xi}^{1}$ iff $\varepsilon(\xi)=1$ iff $\beta \in A_{\xi}^{1}$ iff $\xi \in C_{\beta}$, i.e. $C^{\prime}$ trivializes $\bar{C}$, which is impossible.

ADDED IN PROOF. 1. It has been brought to our attention that during the mid 1970's Laver observed that an $\aleph_{2}$ - $A$-tree with an ascent path is not special, and that, somewhat later, Baumgartner was able to construct such trees from a

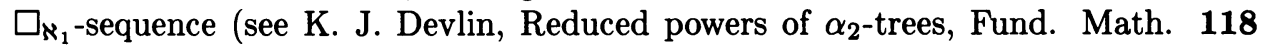
(1983), 129-134) for a presentation of Baumgartner's methods).

2. Concerning Proposition 6.1, Todorevic has recently communicated to us a revision of [11], where, in (1.10), he gives a proof, similar to ours, but considerably terser (he does not go into as much detail about Jensen's construction), that the sequence constructed by Jensen in the proof of Theorem 6.1 of [3], is not trivialized in $V$, assuming that $\kappa$ is not (weakly compact) ${ }^{L}$.

\section{REFERENCES}

1. K. J. Devlin, Constructibility, Springer-Verlag, Berlin and New York, 1984.

2. H. D. Donder, R. B. Jensen, and L. J. Stanley, Condensation-coherent global square systems (A. Nerode et al., eds.), Proc. Sympos. Pure Math., vol. 42, Amer. Math. Soc., Providence, R. I., 1985, pp. 237-258.

3. R. B. Jensen, The fine structure of the constructible hierarchy, Ann. Math. Logic 4 (1972), 229-308.

4. R. Laver and S. Shelah, The $\aleph_{2}$-Souslin-hypothesis, Trans. Amer. Math. Soc. 264 (1981), 411-417.

5. M. Magidor, Reflecting stationary sets, J. Symbolic Logic 47 (1982), 755-771.

6. S. Shelah, Proper forcing, Lecture Notes in Math., vol. 940, Springer-Verlag, Berlin, Heidelberg, and New York, 1982.

7. S. Shelah and L. J. Stanley, S-Forcing. I. A "black box" theorem for morasses, with applications to super-Soslin trees, Israel J. Math. 43 (1982), 185-224.

8. _ Generalized Martin's axiom and Souslin's hypothesis for higher cardinals, Israel J. Math. 43 (1982), 225-236.

9. __ Corrigenda to “Generalized Martin's axiom and Souslin's hypothesis for higher cardinals, Israel J. Math. 53 (1986), 304-314. 
10. F. D. Tall, Some applications of a generalized Martins's axiom (to appear).

11. S. Todorcević, Partitioning pairs of countable ordinals, Acta Math. 159 (1987), 262-294.

12. B. Velickovic, Jensen's $\square$-principles and the Nowák number of partially ordered sets, J. Symbolic Logic 51 (1986), 47-58.

Department of Mathematics, Hebrew University, JeRUSAlem, ISRAel

Department of MATHEMATICS, RUtgers UNIVERSity, NEW BRUNSWICK, NEW JERSEY 08903

Department of Mathematics, Lehigh University, Bethlehem, Pennsylvania 18015

Department of Mathematics, Ohio State University, Columbus, Ohio 43210 